\title{
ADIPONUTRIN LEVELS IN HYPERTENSIVE PATIENTS DEPENDING ON THE DEGREE OF OBESITY
}

\author{
Kravchun P.G., Kadykova O.I., Herasymchuk U.S. \\ Kharkiv National Medical University, Ukraine \\ https://doi.org/10.35339/ic.8.1.10-17
}

\begin{abstract}
Background. It is a well-known the fact that obesity, especially its abdominal (visceral) form, is one of the most important risk factors for hypertension. The purpose. to determine how excessive weight and obesity affect blood serum adiponutrin levels in patients with hypertension. Materials and methods. The study included 58 patients with hypertension. Grade 1 was diagnosed in $12(20.69 \%)$, grade 2 - in $16(27.59 \%)$, grade 3 - in $30(51.72 \%)$ examined patients. Of these, 32 women and 26 men aged 32 to 79 years (mean age $57.5 \pm$ 10.11 years). Patients underwent anthropometric measurements. Blood serum level of glucose was determined by glucose oxidase method, glycosylated hemoglobin was determined by photometric method, total cholesterol, high-density lipoprotein cholesterol, and triglycerides were determined by enzymatic method according to standard biochemical method. The levels of very low density lipoprotein cholesterol, low density lipoprotein cholesterol, atherogenic factor were calculated by the formulas. Adiponutrin blood serum level was determined by enzyme immunoassay method. Results. Adiponutrin blood serum level in all groups of hypertensive patients with overweight and obesity was significantly increased compared to the control group, but the degree of hypertension did not affect the level of adiponutrin. However, the level of the latter in the serum gradually increases according to the duration of hypertension. In addition, the level of adiponutrin increased depending on the degree of obesity, which, in turn, was confirmed by a reliable direct linear relationship between adiponutrin and body mass index. Conclusions. Based on the data obtained, adiponutrin can be considered as a potential biomarker of metabolic disorders.
\end{abstract}

Keywords: adiponutrin, hypertension, obesity, triglycerides.

\section{Introduction}

Hypertension (HT) currently remains an actual problem of cardiology due to high prevalence and is a major risk factor for morbidity and mortality worldwide, resulting in 10.4 million deaths annually [1].

In addition to traditional factors of cardiovascular risk, a complex of factors such as obesity, dyslipidemia, insulin resistance in combination with hypertension attracted considerable attention of scientists. Nowadays, it is generally accepted

\footnotetext{
Corresponding Author:

Uliana Herasymchuk, MD, PhD student of the

Department of Internal Medicine No. 2,

Clinical Immunology and Allergology named

after academician L.T. Malaya., Kharkiv National

Medical University, Kharkiv, Ukraine.

E-mail: ulya.gerasimchuk@gmail.com
}

that the level of increase in the obesity frequency among the population of developed countries is acquiring alarming rates, therefore this phenomenon is compared with a pandemic [2].

It is a well-known the fact that obesity, especially its abdominal (visceral) form, is one of the most important risk factors for HT. According to the World Health Organization (WHO), obesity is defined as «abnormal or excessive fat accumulation that presents a risk to health» $[3,4]$. The actual prevalence of obesity in most European countries is around $20 \%$ [5]. These numbers have nearly tripled since 1986, when the European Association for the Study of Obesity was founded to address the emerging obesity problem [6]. In contrast to the opinion that obesity is only a risk factor for disease, the World Obesity Federation has declared obesity as a chronic recurrent progressive disease [7]. 
Despite the presence of experimental and clinical evidence of the mutual burden HT and obesity, the mechanisms of formation of pathophysiological consequences of such a message remain insufficiently defined. Recently, an important role has been assigned to the adiponutrin (ADPN) protein as a marker for metabolic disorders [8]. ADPN, encoded by gene the patatin-like phospholipase domain-containing 3 (PNPLA3), consists of 481 amino acids responsible for the function of the endoplasmic reticulum, the structure and function of mitochondrial membranes and lipid inclusions in hepatocytes and adipocyte membranes [9]. This protein has hydrolase activity towards triglycerides (TG), acyltransferase activity towards lysophosphatidic acid, and esterase activity towards retinol palmitate [10-12].

The participation of adiponutrin in energy metabolism, triglyceride lipolysis and other metabolic processes has been proven earlier and presented in publications of previous years exclusively within the framework of liver pathology: non-alcoholic fatty liver disease with the risk of coronary heart disease [13], with the risk of chronic kidney disease [14], but data of clinical and experimental studies regarding adiponutrin blood serum level determination in patients with hypertension are very few, and there are almost no scientific data in comorbidity with obesity. Therefore, the study of ADPN - the marker of metabolic disorders to determine the character of the flow of HT in comorbidity with obesity is actually today.

\section{Purpose, subjects and methods:}

2.1. The purpose of the research was to determine how overweight and obesity affect blood serum adiponutrin levels in patients with hypertension.

\subsection{Subjects \& Methods}

The study included 58 patients with HT, who were on inpatient treatment in the cardiology department. Of these, 32 women and 26 men aged 32 to 79 years old (mean age $-57.5 \pm 10.11$ years), who previously have not been receiving regular antihypertensive therapy.

The diagnosis was verified on the basis of clinical, laboratory and instrumental methods of investigation. Exclusion criteria for patients of this study, in addition to patients with symptomatic hypertension, were patients with cancer, acute and chronic inflammatory processes, concomitant diseases of the thyroid gland, and diabetes mellitus. The control group included 20 age-and sexmatched healthy persons. The study was conducted in accordance with the current ethical requirements. The protocol of the study was approved at the meeting of the Committee of Bioethics of the Kharkiv National Medical University, department of Propedeutics of Internal Medicine No. 2 and Nursing Care (Minutes No 7 of 11 September 2018). All patients who participated in the study signed a voluntary informed consent to participate.

All patients had blood pressure (BP) measured in a sitting position after a 5-minute rest according to the Korotkov's method. The guidelines by of European Society of Cardiology (ESC)/ European Society of Hypertension (ESH), criteria of Ukrainian Association of Cardiology were used for verification of the diagnosis and estimation of the hypertension grade [15]. Grade 1 HT was diagnosed in $12(20.69 \%)$ patients, grade $2 \mathrm{HT}-$ $16(27.59 \%)$, and grade 3 HT - $30(51.72 \%)$ examined patients.

The history of increased blood pressure less than 5 years was observed in $27 \%$ of patients, from 6 to 10 years-in 33\%, more than 10 yearsin $40 \%$. Surveys of the examined patients showed the presence of asthenic-neurotic complaints in $98.3 \%$, cerebral complaints $-84.5 \%$, cardiac complaints $-84.5 \%$. It should be noted that in patients with obesity-associated HT, the symptoms were more pronounced. The diagnosis of obesity is established in accordance with the classification based on the determination of body mass index (BMI). This classification is developed by the National Health Institutes by the United States of America, and approved by the WHO. Anthropometric measurements included height $(\mathrm{cm})$, weight $(\mathrm{kg})$, waist circumference $(\mathrm{WC}, \mathrm{cm})$ and hips circumference $(\mathrm{HC}, \mathrm{cm})$. It was followed by calculation of BMI $\left(\mathrm{kg} / \mathrm{m}^{2}\right)$, according to the formula BMI = body weight / height ${ }^{2}$ as well as calculation of the waist to hip ratio (WHR). A WC / $\mathrm{HC}$ value $>0.90$ for male and $>0.85$ for female attesting to the presence of abdominal (visceral) type of adipose tissue distribution. Blood for biochemical research was carried out in the morning on the next day after admission of the patient to the hospital after 1218 hours starvation. Blood was taken from the ulnar vein. All patients were in the same physical activity conditions.

Adiponutrin levels $(\mathrm{pg} / \mathrm{mL})$ were determined by enzyme immunoassay method using The RayBio ${ }^{\circledR}$ Adiponutrin Enzyme Immunoassay (EIA) Kit, (USA).

In order to control carbohydrate metabolism, the glucose level was determined by the fasting 
glucose oxidase method, the content of glycosylated hemoglobin $(\mathrm{HbA} 1 \mathrm{c})$ in blood serum was determined by photometric method by reaction with thiobarbituric acid using a commercial test system of the company «Reagent» (Ukraine). The levels of total cholesterol (TC, mmol/l), highdensity lipoprotein cholesterol (HDL CS, mmol/l) and triglycerides (TG, mmol/l) were determined by enzymatic method using standard kits. The level of very low-density lipoprotein cholesterol (VLDL CS, mmol/l) was calculated using the by the value of the ratio: VLDL CS $=\mathrm{TG} / 2.2$. Low density lipoprotein cholesterol (LDL CS, $\mathrm{mmol} / \mathrm{l}$ ) was determined using the formula Friedewald: LDL CS $=$ TC - (HDL CS + TG/2.2). Coefficient of atherogenicity (CA) was calculated by the Klimov formula: $\mathrm{CA}=(\mathrm{TC}-\mathrm{HDL}$ CS $) /$ HDL CS.

Mathematical computer processing of results was carried out with the help of the software package Statistica 10.0 (StatSoft Inc.). The mean value (Mean) and standard deviation (SD) were determined. Analysis of the data was carried out by methods of nonparametric statistics. In samples with the non-parametric data distribution the results are presented as Me (LQ; UQ), where $\mathrm{Me}$ - median of index, LQ - lower quartile, UQ upper quartile. The Kruskal-Wallis ANOVA, the Mann-Whitney U-test, was used for comparison of the results between groups. Spearman's rank correlation coefficient was used for estimation of the relationship between two variables. The difference in parameters was considered statistically significant at $\mathrm{p}<0.05$.

\section{Results \& Discussion}

During our research, we found a significant increase in ADPN blood serum level in hypertensive patients. The median ADPN in patients of the main group was 5.16 with an interquartile range of 2.78 to $11.33(\mathrm{pg} / \mathrm{mL})$. Compared with the control group, median 1.55 with an interquartile range of 1.37 to $1.68(\mathrm{pg} / \mathrm{mL}$ ) (Fig. 1).

The patients were divided into 3 groups depending of the degree of HT. To identify differences when comparing these groups using the Kruskal-Wallis ANOVA, statistically significant differences were found $(\mathrm{H}=36.58$; $\mathrm{p}<0.001)$. For a more accurate description of the differences in the groups, they were compared in pairs, taking into account the median.

The level of the studied ADPN in all groups of patients with HT was found to be significantly increased compared with the control group: 8.64 [2.94 - 10.60]- $3.81[1.72-7.85]-8.14$ [3.12$12.00]$ and 1.55 [ $1.37-1.68](\mathrm{pg} / \mathrm{mL})(\mathrm{p}<$ 0.001 ), respectively to HT 1 group, HT 2 group, HT 3 group and control group. As seen from the Fig. 2, the level of the latter in the blood serum does not depend on the grade of HT. When studying the effect of the duration of the increase in blood pressure on the level of ADPN found that $\mathrm{H}=7.28 ; \mathrm{p}=0.028$. Pairwise comparison of groups, which is shown in Fig. 3, shows that the level of ADPN is higher in patients with the duration of HT of more than 5 years and was significantly increased compared with the hypertensive patients lasting up to 5 years group:

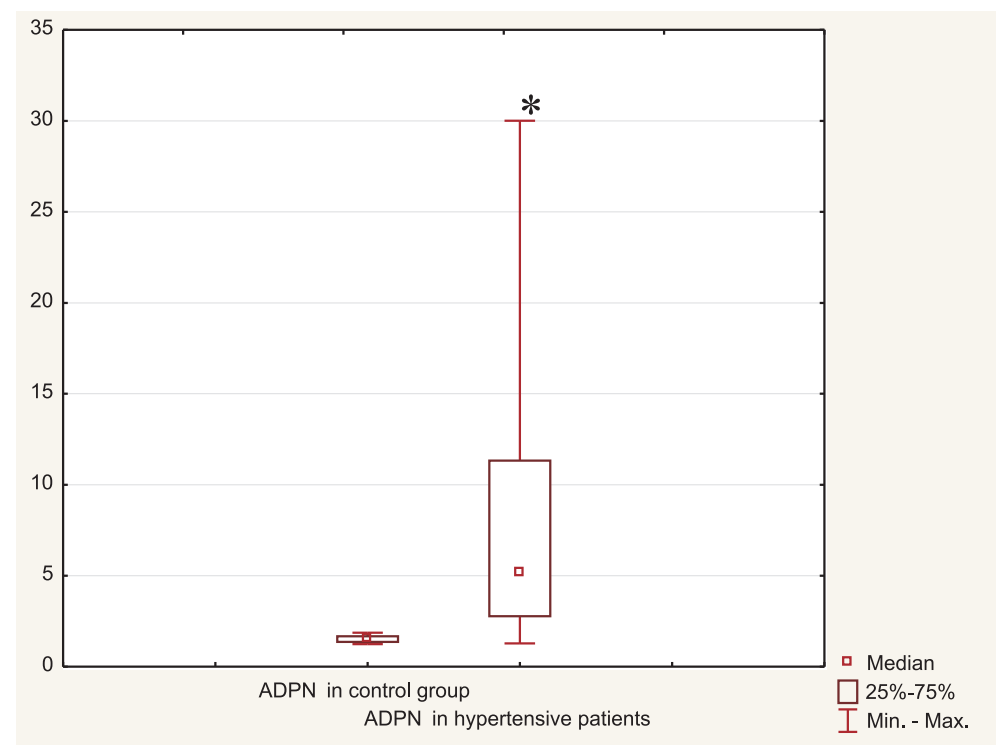

Fig. 1 The level of ADPN ( $\mathrm{pg} / \mathrm{mL})$ in control group and hypertensive patients. Note: $*$ the difference is statistically significant compared with control group $(\mathrm{p}<0,05)$ 


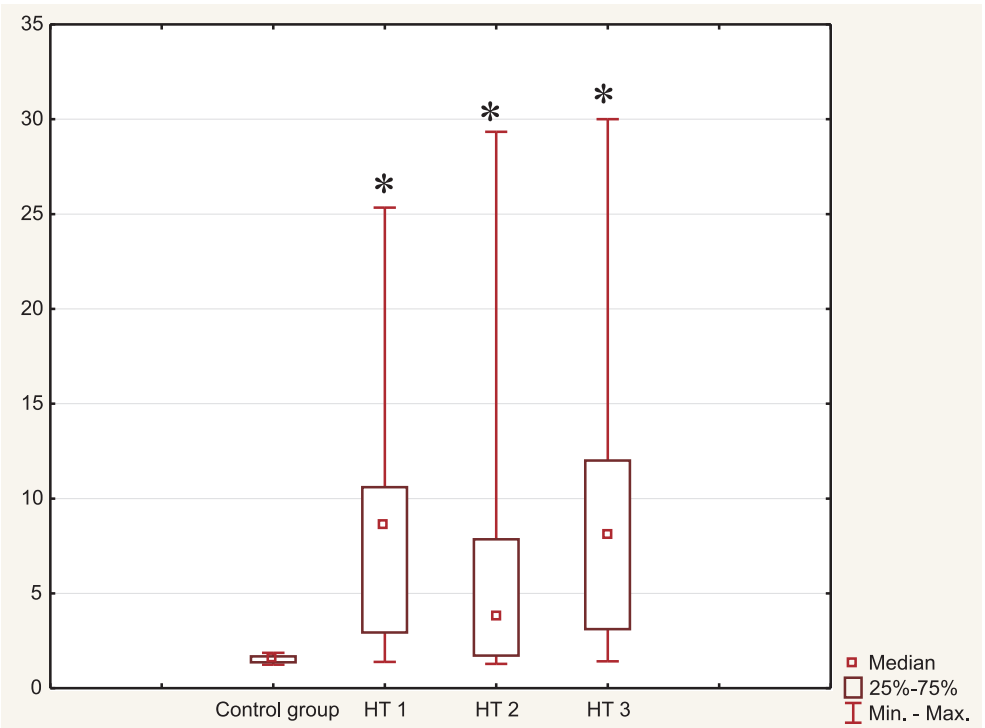

Fig. 2. The level of ADPN (pg/mL) depending of the grades of HT Note: * the difference is statistically significant compared with control group $(\mathrm{p}<0,001)$

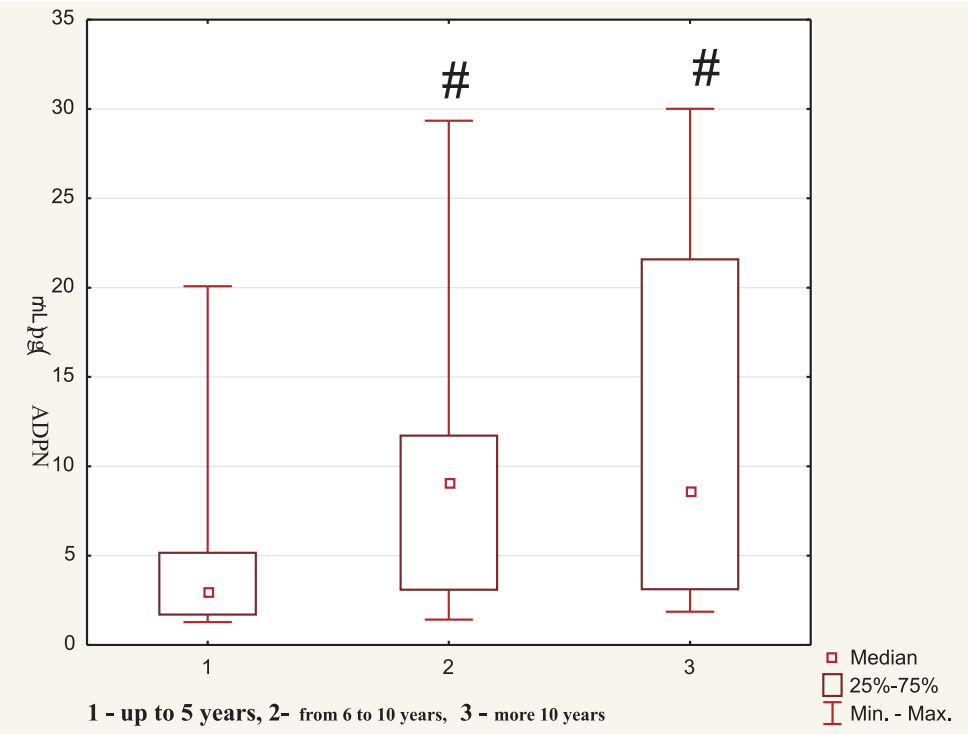

Fig. 3. The level of ADPN (pg/mL) in hypertensive patients depending on the duration of hypertension, Me [LQ; UQ]

Note: "the difference is statistically significant compared with 1 group $(\mathrm{p}<0,05)$

$9.03[3.09-11.72]-8.63[3.12-21.58]-2.96$ [1.71-5.16] $(\mathrm{pg} / \mathrm{mL})(\mathrm{p}<0.05)$, respectively to hypertensive patients lasting from 6 to 10 years group, hypertensive patients lasting more 10 years group and hypertensive patients lasting up to 5 years group.

To analyze the effect of the level of increase in body weight for the the level of ADPN, groups of patients with obesity and without obesity were identified. When comparing these groups using the Kruskal-Wallis test, statistically significant differences were found $(\mathrm{H}=56.29 ; \mathrm{p}<0.001)$. For a more accurate description of the differences in the groups, they were compared in pairs, taking into account the median (Table 1). As seen from the Table 1, the indices of the concentration of the latter in the blood serum significantly differed between the control group in comparison with the patients of the above groups, as well as directly and in the groups among themselves $(\mathrm{p}<0.001)$. At the same time, in obese patients, the median of 10.45 [5.08-20.08] ( $\mathrm{pg} / \mathrm{mL})$ had the greatest value compared to the group of patients without obesity, 2.03 [1.68-3.09] (pg/mL).

In order to study the effect of the degree of obesity on the concentration of ADPN, both in the group of obese and non-obese patients, subgroups were identified depending of the body 
Table 1

$A D P N$ level in hypertensive patients depending of the level of increase in body weight and in the control group, Me (LQ; UQ)

\begin{tabular}{|c|c|c|c|c|}
\hline Index & $\begin{array}{c}\text { Control group } \\
(\mathrm{n}=20)\end{array}$ & $\begin{array}{c}\text { Hypertensive patients } \\
\text { without obesity } \\
(\mathrm{n}=19)\end{array}$ & $\begin{array}{c}\text { Hypertensive patients } \\
\text { with obesity } \\
(\mathrm{n}=39)\end{array}$ & $\begin{array}{c}\mathrm{P} \\
(\text { Mann-Whitney } \\
\text { U-test })\end{array}$ \\
\hline ADPN $(\mathrm{pg} / \mathrm{mL})$ & 1.55 & 2.03 & 10.45 & $\begin{array}{c}\mathrm{p}_{0-1}<0.001 \\
\mathrm{p}_{0-2}<0.001 \\
\end{array}$ \\
& {$[1.37-1.68]$} & {$[1.68-3.09]$} & {$[5.08-20.08]$} & $\mathrm{p}_{1-2}<0.001$ \\
\hline
\end{tabular}

mass index. When comparing these groups using the Kruskal-Wallis test, statistically significant differences were found $(\mathrm{H}=68.27 ; \mathrm{p}<0.001)$. To find out which groups differed among themselves, they were pairwise compared with the median taken into account. We see that the level of ADPN in all of the above subgroups of patients was significantly increased compared to the control group: 3.09 [2.78-3.22] - $4.42[3.85-8.63]-$ $10.86[10.45-11.72]-27.11[24.72-29.13]-1.55$ $[1.37-1.68]-(\mathrm{pg} / \mathrm{mL})(\mathrm{p}<0.05)$, respectively to overweight group, grade 1 obesity group, grade 2 obesity group, grade 3 obesity group and control group, excluding the subgroups of patients with normal body weight group - 1.71 [1.42-1.94] ( $>0.05$ ).

As seen from the Fig. 4, the level of the latter in the blood serum gradually increases according to the degree of obesity, and the maximum level of ADPN was noted in the subgroup of patients with obesity of the third degree.
To identify the relationship between ADPN and other indicators, a correlation analysis was carried out (Table 2). There were no significant correlations with systolic blood pressure (SBP), diastolic blood pressure (DBP), heartbeats (HB), indicators of the carbohydrate profile, lipid profile, with the exception of VLDL CS and TG. Namely, a reliable direct linear relationship between ADPN and VLDL CS $(\mathrm{r}=0.34, \mathrm{p}<0.05)$ and a reliable direct linear relationship between ADPN and TG $(r=0.35, p<0.05)$ in both cases, the strength of the bond was moderate. Also, a significant direct linear relationship between ADPN was noted with WS, HC, WHR, body weight, and noteworthy with a BMI $(r=0.90, p<0.05)$, which has very high character.

There are a large number of hypothetical pathogenic mechanisms through which obesity can lead to HT. They include the activation of the sympathetic nervous system, the renin-angiotensinaldosterone system, metabolic disorders (including

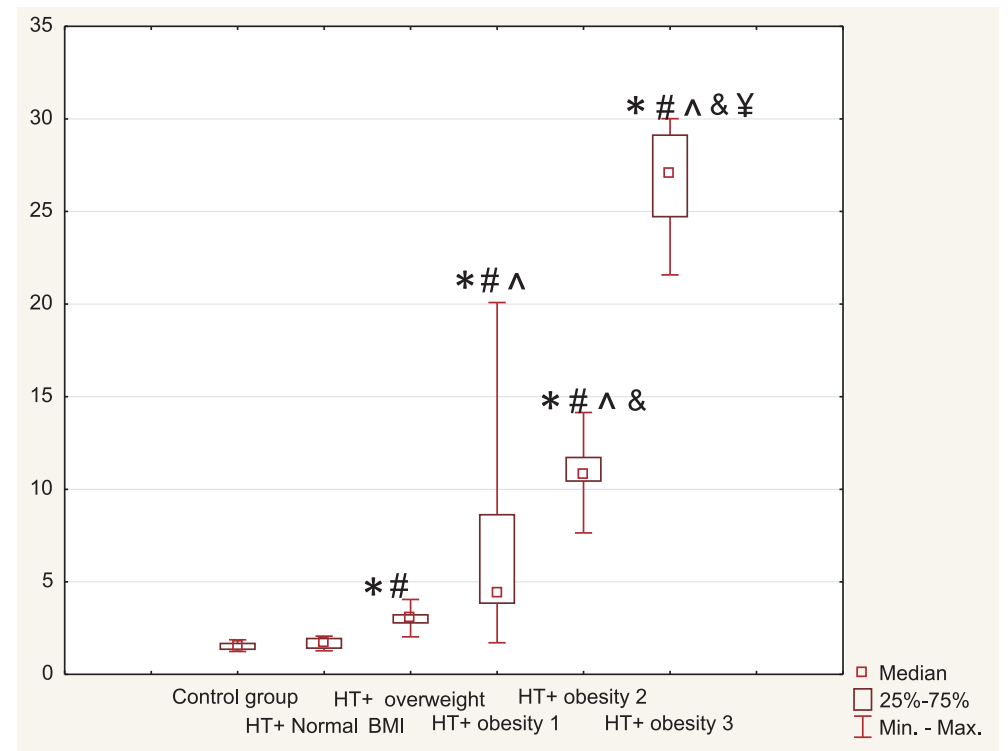

Fig. 4 ADPN level (pg/mL) depending from the presence and degree of obesity

Note: $*$ - the difference is statistically significant compared with control group $(\mathrm{p}<0,05)$,

$\#$ - the difference is statistically significant compared with HT+ Normal BMI group $(\mathrm{p}<0,05)$,

$\wedge$ - the difference is statistically significant compared with HT+ overweight group $(\mathrm{p}<0,05)$,

$\&$ - the difference is statistically significant compared with HT+ obesity 1 group $(\mathrm{p}<0,05)$,

$\Gamma-$ the difference is statistically significant compared with HT+ obesity 2 group $(\mathrm{p}<0,05)$. 
Table 2

Correlation links between $A D P N$ and $S B P, D B P, H B$, parameters of carbohydrate and lipid metabolism, BMI and anthropometric parameters of patients $(n=58)$

\begin{tabular}{|c|c|c|}
\hline Index & $\mathbf{r}$ & $\mathbf{p}$ \\
\hline $\mathrm{SBP}, \mathrm{mmHg}$ & 0.20 & 0.14 \\
\hline $\mathrm{DBP}, \mathrm{mmHg}$ & 0.15 & 0.27 \\
\hline $\mathrm{HB}$, beats per minute & 0.09 & 0.48 \\
\hline Glucose, $\mathrm{mmol} / \mathrm{L}$ & 0.16 & 0.22 \\
\hline $\mathrm{HbA}_{1 \mathrm{c}}$ & 0.17 & 0.19 \\
\hline HDL CS mmol/L & -0.03 & 0.83 \\
\hline LDL CS mmol/L & -0.09 & 0.50 \\
\hline VLDL CS mmol/L & 0.34 & 0.009 \\
\hline TC mmol/L & -0.07 & 0.62 \\
\hline CA & -0.11 & 0.40 \\
\hline TГ $\mathrm{mmol} / \mathrm{L}$ & 0.35 & 0.007 \\
\hline BMI & 0.90 & 0.001 \\
\hline WC, $\mathrm{cm}$ & 0.80 & 0.001 \\
\hline $\mathrm{HC}, \mathrm{cm}$ & 0.55 & 0.001 \\
\hline WHR & 0.65 & 0.001 \\
\hline Weight, kg & 0.86 & 0.001 \\
\hline
\end{tabular}

hyperinsulinemia, imbalance of adipokines, an increase in the number of cytokines). The influence of adipokines, in particular ADPN, in conditions of overweight and obesity, is possibly one of the key processes in the development of hypertension. Here it is necessary to take into account the general effect of the imbalance of adipokines, their maintenance of oxidative stress and inflammation, which leads to endothelial dysfunction.

According to most literature assumptions, ADPN, encoded by gene the patatin-like phospholipase domain-containing 3 (PNPLA3), is a liver-specific human protein $[16,17]$. In particular, in studies by Johansson L.E., in 2009 it was shown that the G-allele of the PNPLA3 gene positively correlates with the content of triglycerides in liver tissue [18]. But Qadri S. et al. concluded that this protein is found not only in the human liver, but also in large quantities in the abdominal tissue, and in particular human abdominal tissue to contain approximately nine-fold more PNPLA3 protein than the liver at the level of the whole body, which contradicts most previous assumptions [19]. Excessive accumulation of TG is a hallmark of obesity. Fatty acids released during hepatic TG hydrolysis can be used for $\beta$-oxidation, signaling, and for VLDL-CS synthesis. Lipolysis was initially thought to be concentrated in adipose tissue and catalyzed by only two lipases, hormone-sensitive lipase (HSL) and monoacylglycerol lipase. However, genetic elimination of HSL expression in mice failed to eliminate TG hydrolysis in adipocytes, leading to the identification of a third lipase termed adipose triglyceride lipase (ATGL). Although these three enzymes are considered major players that regulate lipolysis in adipocytes, other lipolytic enzymes that promote TG metabolism in the liver have been described. One of them is ADPN [20].

In our study, patients with hypertension with overweight and obesity showed a significant increase in adiponutrin blood serum level compared with the control group. $(5.16 \mathrm{pg} / \mathrm{mL}$ vs. $1.55 \mathrm{pg} / \mathrm{mL}$, $\mathrm{p}<0.05)$. It was estimated that blood serum adiponutrin levels depend on the duration of hypertension. It was estimated that the level of adiponutrin in blood serum depends on the body mass index, which is confirmed by a reliable direct linear relationship $(r=0,90 ; p<0,001)$.

Based on the data obtained, adiponutrin can be considered as a potential biomarker of metabolic disorders. It is important to conduct further studies to evaluate the activity of adiponutrine with a longer observation period in broader populations. The results of new studies will help to study the pathogenetic mechanisms of this comorbidity, namely hypertension with obesity and its use in clinical practice as a potential biomarker of metabolic disorders.

\section{Conclusions}

- Hypertensive patients with overweight and obesity showed a significant increase in adiponutrin blood serum level compared with the control group.

- It was estimated that blood serum adiponutrin levels depend on the duration of hypertension.

- It was estimated that the level of adiponutrin in blood serum depends on the body mass index, which is confirmed by a reliable direct linear relationship.

\section{Conflict of interests}

The authors of the article declare no conflict of interests. 


\section{References}

1. GBD 2017 Risk Factor Collaborators. (2018). Global, regional, and national comparative risk assessment of 84 behavioural, environmental and occupational, and metabolic risks or clusters of risks for 195 countries and territories, 1990-2017: a systematic analysis for the Global Burden of Disease Study 2017. Lancet (London, England), 392(10159), 1923. doi: 10.1016/S0140-6736(18)32225-6.

2. Afshin, A., Forouzanfar, M. H., Sur, P., Estep, K., \& Lee, A. (2017). Health effects of overweight and obesity in 195 countries over 25 years. GBD 2015 Obesity Collaborators. N Engl J Med, 377, 13-27. doi: 10.1056/NEJMoa1614362.

3. WHO. World Health Organization. 2016. ProMED-mail website. Retrieved from http://: www.who.int/mediacentre/factsheets/fs311/en/.

4. Bluher, M. (2020). Metabolically healthy obesity. Endocrine Reviews, 41(3), doi: 10.1210/endrev/ bnaa004.

5. Yumuk, V., Tsigos, C., Fried, M., Schindler, K., Busetto, L., Micic, D., Toplak, H. (2015). European guidelines for obesity management in adults. Obesity facts, 8(6), 402-424. doi: 10.1159/000442721.

6. Hauner, H., Woodward, E. (2016). Looking Back-EASO Is Celebrating Its 30th Anniversary. Obesity facts, 9(5), 363-364. doi: 10.1159/000452647.

7. Bray, G. A., Kim, K. K., Wilding, J. P. H., on behalf of the World Obesity Federation. (2017). Obesity: a chronic relapsing progressive disease process. A position statement of the World Obesity Federation. Obesity Reviews, 18(7), 715-723. doi: 10.1111/obr.12551.

8. Winberg, M. E., Motlagh, M. K., Stenkula, K. G., Holm, C., Jones, H. A. (2014). Adiponutrin: a multimeric plasma protein. Biochemical and biophysical research communications, 446(4), 1114-1119. doi: 10.1016/j.bbrc.2014.03.078.

9. Huang, Y., He, S., Li, J. Z., Seo, Y. K., Osborne, T. F., Cohen, J. C., Hobbs, H. H. (2010). A feedforward loop amplifies nutritional regulation of PNPLA3. Proceedings of the National Academy of Sciences, 107(17), 7892-7897. doi: 10.1073/pnas.1003585107.

10. Pingitore, P., Pirazzi, C., Mancina, R. M., Motta, B. M., Indiveri, C., Pujia, A., Montalcini, T., Hedfalk, K., Romeo, S. (2014). Recombinant PNPLA3 protein shows triglyceride hydrolase activity and its I148M mutation results in loss of function. Biochimica et Biophysica Acta (BBA)-Molecular and Cell Biology of Lipids, 1841(4), 574-580. doi: 10.1016/j.bbalip.2013.12.006.

11. Pirazzi, C., Valenti, L., Motta, B. M., Pingitore, P., Hedfalk, K., Mancina, R. M., ... \& Maglio, C. (2014). PNPLA3 has retinyl-palmitate lipase activity in human hepatic stellate cells. Human molecular genetics, 23(15), 4077-4085. doi: 10.1093/hmg/ddu121.

12. Kienesberger, P. C., Oberer, M., Lass, A., Zechner, R. (2009). Mammalian patatin domain containing proteins: a family with diverse lipolytic activities involved in multiple biological functions. Journal of lipid research, 50 (Supplement), 63-68. doi: 10.1194/jlr.R800082-JLR200

13. Wu, J. T., Liu, S. S., Xie, X. J., Liu, Q., Xin, Y. N., Xuan, S. Y. (2020). Independent and joint correlation of PNPLA3 I148M and TM6SF2 E167K variants with the risk of coronary heart disease in patients with non-alcoholic fatty liver disease. Lipids in Health and Disease, 19(1), 1-7. doi: 10.1186/ s12944-020-01207-9.

14. Mantovani, A., Chiara, Z. (2020). PNPLA3 gene and kidney disease. Exploration of Medicine, 1 , 42-50. doi: 10.37349/emed.2020.00004.

15. Williams, B., Mancia, G., Spiering, W., Agabiti Rosei, E., Azizi, M., Burnier, M.,\& Kahan, T. (2018). 2018 ESC/ESH Guidelines for the management of arterial hypertension: The Task Force for the management of arterial hypertension of the European Society of Cardiology (ESC) and the European Society of Hypertension (ESH). European Heart Journal, 39(33), 3021-3104. doi: 10.1093/eurheartj/ ehy339.

16. Trepo, E., Romeo, S., Zucman-Rossi, J., \& Nahon, P. (2016). PNPLA3 gene in liver diseases. Journal of hepatology, 65(2), 399-412. doi: 10.1016/j.jhep.2016.03.011.

17. Bruschi, F. V., Claudel, T., Tardelli, M., Caligiuri, A., Stulnig, T. M., Marra, F., Trauner, M. (2017). The PNPLA3 I148M variant modulates the fibrogenic phenotype of human hepatic stellate cells. Hepatology, 65(6), 1875-1890. doi: 10.1002/hep.29041.

18. Johansson, L. E., Johansson, L. M., Danielsson, P., Norgren, S., Johansson, S., Marcus, C., Ridderstrale, M. (2009). Genetic variance in the adiponutrin gene family and childhood obesity. PLoS One, 4(4). doi: 10.1371/journal.pone.0005327. 
19. Qadri, S., Lallukka-Bruck, S., Luukkonen, P. K., Zhou, Y., Gastaldelli, A., Orho-Melander, M. \& Hakkarainen, A. (2020). The PNPLA3?I148M variant increases polyunsaturated triglycerides in human adipose tissue. Liver International, 40(9), 2128-2138. doi: 10.1111/liv.14507.

20. Quiroga, A. D., Lehner, R. (2018). Pharmacological intervention of liver triacylglycerol lipolysis: The good, the bad and the ugly. Biochemical pharmacology, 155, 233-241. doi: 10.1016/j.bcp.2018.07.005.

Received: 22-Dec-2020

Accepted: 03-Mar-2021 